\title{
Demanda Cognitiva de Estándares Educativos y Libros de Texto para la Enseñanza del Álgebra en Honduras
}

\section{Cognitive Demand for Educational Standards and Textbooks for Algebra Teaching in Honduras}

\author{
Luis Armando Ramos Palacios* \\ ORCID iD 0000-0002-8532-9862 \\ Luis Manuel Casas García** \\ ORCID iD 0000-0002-2752-1784
}

\begin{abstract}
Resumen
Este trabajo está orientado a una valoración del alineamiento o coherencia, a nivel de demanda cognitiva, entre los estándares educativos y las tareas o actividades que proponen los libros de texto oficiales para la enseñanza de las Matemáticas para el bloque de Álgebra en los grados $7^{\circ}, 8^{\circ}$ y $9^{\circ}$ de la Educación Básica en Honduras. Si se asegura un verdadero alineamiento entre los estándares educativos, los libros de texto y las evaluaciones, se ayuda a mejorar los procesos de implementación del currículo, a valorar con mejor criterio los resultados de las evaluaciones y a involucrar a los docentes en los procesos de mejora. Con la ayuda de veinte docentes de Matemáticas, hondureños, con amplia experiencia en estos grados y aplicando el modelo propuesto por Webb (1997), se clasificaron, según el nivel de demanda cognitiva, treinta estándares del boque de Álgebra y 110 preguntas de las pruebas nacionales de evaluación. A partir de esta clasificación fueron evaluadas, posteriormente, 1912 tareas similares a las propuestas en dichas pruebas y recogidas en los libros de texto oficiales para los grados en estudio. Los resultados encontrados indican que no existe un adecuado alineamiento a nivel de demanda cognitiva, en el bloque de Álgebra, entre estándares educativos y actividades propuestas en los libros de texto. Consideramos que la metodología utilizada puede resultar útil para extenderla a otros bloques de Matemáticas, así como a otras áreas de la educación básica.
\end{abstract}

Palabras Clave: Alineamiento. Estándares Educativos. Libros de Texto. Demanda Cognitiva. Álgebra.

\begin{abstract}
This work is oriented to assess the alignment or coherence, in a cognitive demand level; between the educational standards and the tasks or activities proposed by official textbooks, for teaching mathematics within 7 th, 8 th and 9th grades in basic education in Honduras. If an authentic alignment between the educational standards, textbooks and the evaluations is ensured, this shall be helpful in the improvement of curriculum implementation processes; it asses in a more realistic way the results of evaluations and come out with a higher degree of assertion in the elements that require attention and make teachers part of the improvement process. With the help of twenty Math Honduran teachers, with vast experience in these grades and, applying the Webb alignment model criterion we classified, according to the level of cognitive demand, thirty standards of the algebra block and 110 questions or

\footnotetext{
* Doctor en Educación por la Universidad de Extremadura (UEx), España. Profesor Titular del Departamento de Matemáticas de la Universidad Pedagógica Nacional Francisco Morazán (UPNFM), Tegucigalpa, Honduras. Dirección postal: Colonia el Dorado, frente a Plaza Miraflores Tegucigalpa, Honduras. E-mail: luramosp@upnfm.edu.hn.

** Doctor en Psicopedagogía por la Universidad de Extremadura (UEx), España. Profesor Titular de Universidad en el área de Didáctica de las Matemáticas en la Universidad de Extremadura, España. Dirección postal: Avda. de Elvas, s/n, Badajoz, España, C.P: 06071. E-mail: luisma@unex.es.
} 
items of the national evaluation tests. This classification worked as a reference to sort 1912 algebra tasks or activities proposed by official textbooks and standards for teaching math in the studied grades. The results show that there is no adequate alignment or coherence at the level of cognitive demand in the algebra block between these two pieces of the curriculum: Education Standards and Textbooks in the third cycle of basic education in Honduras. We consider that the methodology used can be useful to extend it to other math blocks, as well as to other areas of basic education.

Keywords: Alignment. Educational Standards. Textbooks. Cognitive Demand. Algebra.

\section{Introducción}

La enseñanza aprendizaje de las Matemáticas en el sistema educativo hondureño ha sido, desde inicios del presente siglo, un tema en la agenda de la Secretaría de Educación del país.

Se han impulsado reformas educativas como respuesta a los compromisos asumidos por el gobierno de Honduras como parte del programa educación para todos, conocido por sus siglas en inglés como plan EFA (Education For All) el cual propuso varias metas que cumplir en el período 2003 a 2015, siendo una de ellas la de incrementar el rendimiento académico en los alumnos de sexto grado en Matemáticas y Español (EDUCACIÓN, 2003).

El Sistema Educativo Nacional de Honduras está organizado en tres niveles: Educación Preescolar, con una duración de tres años (niños de 3 a 6 años de edad), Educación Básica (de 7 a 15 años), organizada en tres ciclos de tres años cada uno e incluye los grados de $1^{\circ}$ a $9^{\circ}$ y Educación Media que se refiere a los Bachilleratos (jóvenes de 16 a 18 años).

Una de las primeras tareas fue la de desarrollar el Currículo Nacional para Educación Básica (CNEB) que establece, de manera detallada, contenidos conceptuales, actitudinales y procedimentales de cada área del conocimiento que deben ser abordados en los grados de $1^{\circ} \mathrm{a}$ $9^{\circ}$.

El CNEB organiza la enseñanza de las Matemáticas en cinco bloques de contenido: Números y Operaciones, Geometría, Medidas, Álgebra y Estadística, los cuales son abordados a lo largo de los nueve grados.

En la búsqueda de la implementación del CNEB en todos los centros educativos públicos del país y el logro de las metas EFA, la Secretaría de Educación de Honduras tuvo el apoyo de la cooperación internacional a través de proyectos que financiaron la construcción de recursos pedagógicos, orientados principalmente a las áreas de Español y Matemáticas, tales como estándares educativos nacionales y libros de texto, que incluyen guías metodológicas para el maestro y cuadernos de trabajo para los alumnos.

De acuerdo con lo expresado por Carr y Harris (2001) es indudable que la disponibilidad 
y uso adecuado de materiales educativos de apoyo a la labor docente implican mejoras en el aprovechamiento escolar pero el cambio exitoso ocurre cuando todos los aspectos del currículo están alineados a los estándares a través de un sistema coherente y propositivo de procesos y productos. Los libros de texto son utilizados para la enseñanza de las Matemáticas de $1^{\circ}$ a $9^{\circ}$ grado y los estándares educativos nacionales, además de ser un referente para la labor docente, orientan las evaluaciones nacionales con el propósito de valorar el cumplimiento de las metas educativas.

Los informes de rendimiento académico de los últimos seis años, generados por las evaluaciones nacionales que realiza la Secretaría de Educación, muestran resultados altamente preocupantes en el área de Matemáticas. En el tercer ciclo, menos del 10\% de los estudiantes demuestran un rendimiento académico satisfactorio (EDUCACIÓN, 2017), lo cual es particularmente preocupante en el bloque de Álgebra. Realizar un proceso de análisis y evaluación de la coherencia entre los elementos del currículo es imprescindible para asegurar que estos elementos buscan alcanzar los mismos objetivos.

Considerando que unos mismos libros de texto oficiales son empleados, de forma general, en todos los centros educativos públicos, y que estos recursos pedagógicos tienen gran influencia en el proceso enseñanza aprendizaje de las Matemáticas, la pregunta que nos planteamos es: ¿La demanda cognitiva de los contenidos algebraicos que presentan los libros de texto se corresponden con lo que exigen los estándares educativos? Esta investigación tiene como objetivo valorar el grado de alineamiento, a nivel de demanda cognitiva, entre lo que debe enseñarse, según lo recogido en los estándares educativos y lo que realmente se enseña en los libros de texto.

Para hacer esta valoración de alineamiento adaptamos el modelo de Webb (1997) diseñado, en su origen, para analizar el alineamiento entre estándares y evaluación. La adaptación del modelo requiere analizar el nivel de demanda cognitiva de los contenidos de los estándares y el de las tareas o actividades que proponen los libros de texto.

Consideramos que los objetivos del estudio son de interés, pues en base a sus resultados se espera ofrecer información que permita evaluar la calidad de estos recursos de cara a posibles reformas futuras.

\section{Marco Teórico}

\subsection{Estándares Educativos Nacionales}


La tendencia de organizar la educación matemática en función de estándares surge a finales de los años 80, en Estados Unidos. El Comité Nacional de Profesores de Matemáticas (NCTM, por sus siglas en inglés) asumió el compromiso de dar una respuesta a partir de algunos principios y estándares que sirviesen como un marco de referencia o base que orientase la educación matemática en el ámbito escolar, con la misión de proveer una visión y dirección necesaria para una Educación Matemática de alta calidad para todos los estudiantes (NCTM, 2000).

En el ámbito hondureño, los estándares están definidos como objetivos educativos que señalan, con claridad, lo que los alumnos tienen que aprender y ser capaces de saber y saber hacer (EDUCACIÓN, 2011a) y fueron diseñados con la idea de que sirviesen de guía y orientasen la labor educativa en todos los centros educativos del país, y las evaluaciones de rendimiento académico.

Los estándares educativos para el área de Matemáticas están organizados de acuerdo a los bloques de contenido que indica el CNEB. Cada bloque tiene componentes, y cada componente tiene sus correspondientes estándares.

Por lo que se refiere al bloque de Álgebra, incluye los siguientes componentes: Comparación y orden, Ecuaciones y desigualdades, Posición, Expresiones algebraicas, Razones y proporciones y Funciones y cada componente tiene asociado objetivos específicos o estándares educativos, como se muestra en los cuadros 2, 3 y 4 (Anexo A).

Como podemos observar, es trascendental la importancia del estudio del Álgebra en el contexto escolar, lo que tratamos de cuestionar es si la exigencia cognitiva que supone cada estándar se corresponde con la que presentan los libros de texto utilizados para enseñar Matemáticas en el tercer ciclo.

La Figura 1 muestra, según el número de estándares relacionados con cada bloque de contenidos del currículum de Matemáticas, la cobertura que le dedican lo largo de los distintos grados. Por ejemplo, el bloque Números y Operaciones tiene mayor cobertura en $7^{\circ}$ que en los otros dos grados, mientras que el bloque de Álgebra tiene un mayor énfasis en $9^{\circ}$. 


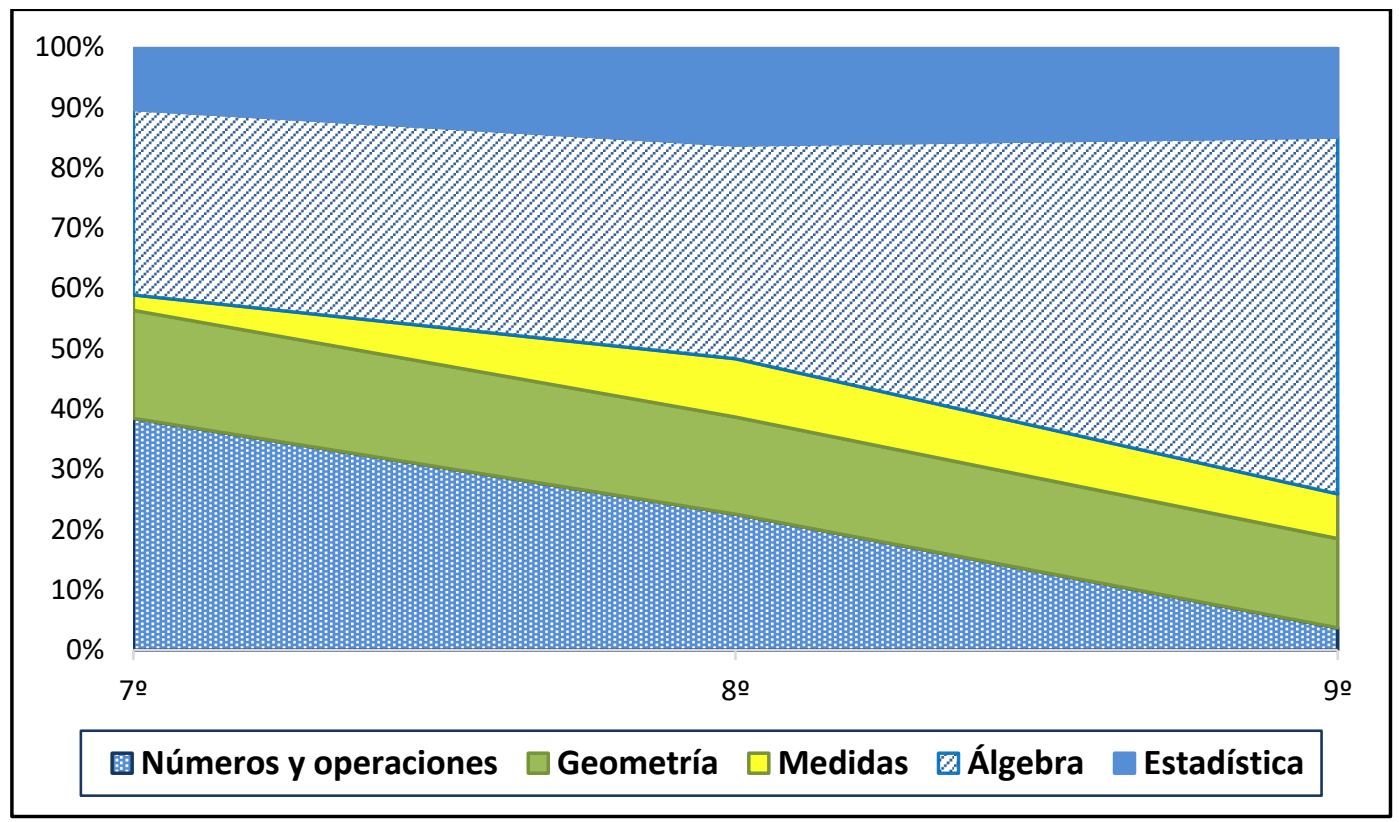

Figura 1 - Cobertura de los estándares de Matemáticas por los bloques

Fuente: Elaboración propia a partir de los estándares educativos nacionales (EDUCACIÓN, 2011a).

\subsection{Libros de Texto}

Uno de los elementos de mayor influencia en el proceso de enseñanza aprendizaje de las Matemáticas son los libros de texto utilizados, y merecen atención y análisis exhaustivo con la idea de asegurar que corresponden a lo que verdaderamente exige el currículo. En este sentido, Llinares (2008) justifica las investigaciones sobre el papel de los libros de texto, al afirmar que la manera en que el contenido matemático es organizado y considerado en los libros de texto puede tener una influencia determinante sobre la práctica del profesor y sobre los procesos de enseñanza aprendizaje generados en las aulas, de los que forman parte las evaluaciones internas y externas.

Los libros de texto, como afirman Martínez y Rodríguez (2010), siguen siendo, en plena era digital, el dispositivo didáctico que hegemoniza el desarrollo curricular en las aulas de primaria, secundaria y bachillerato y, con intensidad creciente, también en las aulas de infantil. Son una herramienta básica para que los docentes puedan implementar el currículo, para que los alumnos tengan facilidades de aprender los contenidos y, sobre todo, que sirvan de guía para evaluar el logro de los objetivos propuestos.

Los textos escolares son herramientas que influyen en el proceso enseñanza aprendizaje y juegan un papel aún más preponderante en los países en vías de desarrollo, donde, además, actúan como una herramienta de desarrollo en la profesionalización docente (LEBRUN, MORESOLI; HASNI, 2012). 
En el caso de Honduras, varios procesos de capacitación docente realizados en los últimos años, en relación con la enseñanza aprendizaje de las Matemáticas, se han centrado en el uso y manejo de los libros de texto, los cuales, además de brindar orientaciones pedagógicas, han servido para fortalecer los conocimientos matemáticos básicos de los docentes en servicio.

El proyecto encargado del diseño de los libros de texto oficiales en Honduras, llamado Proyecto Mejoramiento en la Enseñanza Técnica en el Área de Matemáticas (PROMETAM), los define como los documentos acordes con los requerimientos técnico pedagógicos que se necesitan para la enseñanza y el aprendizaje de las Matemáticas, orientados en el Diseño del Currículo Nacional Básico de Honduras, con el fin de crear las condiciones necesarias para que los docentes puedan desarrollar mejor su trabajo y los niños y jóvenes puedan aprender mejor los contenidos matemáticos (JICA, 2015).

Los libros de texto oficiales lo forman un cuaderno de trabajo para el alumno y una guía del docente que presenta indicaciones metodológicas para abordar cada uno de los temas, y son utilizados en todos los centros públicos del país.

\subsection{Demanda cognitiva y alineamiento}

La demanda cognitiva o profundidad de conocimiento (Depth-of-Knowledge) también conocida como DOK (por sus siglas en inglés), constituye una forma de clasificar el aprendizaje por niveles de profundidad de conocimiento, considerando lo que es capaz de hacer el estudiante e integrando criterios sobre niveles de pensamiento orientados a procesos de conocimiento, memoria, comprensión, aplicación, análisis, síntesis y evaluación.

Para Penalva y Llinares (2011) lo que la tarea exige a los estudiantes determina la demanda cognitiva de la tarea y en parte su potencial de aprendizaje. De esta manera, definen la demanda cognitiva de una tarea como la clase y nivel de pensamiento que su resolución exige a los alumnos. La clase y nivel de pensamiento en el que se implican a los estudiantes determinará lo que ellos pueden llegar a aprender. Así, las tareas se tienen que corresponder con los objetivos de aprendizaje y llegar a reflejar un amplio rango de ellos (PENALVA; LLINARES, 2011).

Valorar la demanda cognitiva de una tarea o actividad implica conocer el esfuerzo mental que supone resolverla con éxito y una manera de valorarla es clasificarla de acuerdo con cierto modelo. Conocer la demanda cognitiva de una tarea y de los respectivos estándares educativos es una etapa del proceso de evaluación del alineamiento o coherencia que existe entre ellos, independientemente del modelo que se utilice para evaluarlo. 
El concepto de alineamiento se conoce desde hace algún tiempo, y ganó más prominencia en la década de 1990, con el advenimiento de los estándares y los movimientos de reformas educativas en Estados Unidos de América (WEBB, 2002).

El modelo de Norman L. Webb, conocido como modelo Webb, se ha utilizado para valorar el alineamiento entre los estándares y las evaluaciones en distintas áreas curriculares como Lenguaje, Matemáticas, Estudios Sociales y Ciencias, en muchos distritos escolares en Estados Unidos, y es considerado como uno de los pioneros en los trabajos de alineamiento entre currículo y evaluación (HERMAN; WEBB; ZUNIGA, 2005).

La utilización del modelo Webb para evaluar el grado de alineamiento entre estándares educativos y evaluación supone un estudio de tipo cuantitativo (MARTONE; SIRECI, 2009) que busca, a través de indicadores numéricos, determinar el grado de alineamiento a nivel de demanda cognitiva entre ellos.

La aplicación del modelo Webb, que para el presente trabajo hemos utilizado con el fin de evaluar el alineamiento entre estándares y libros de texto, supone la clasificación de los estándares educativos y las tareas que propone el libro de texto de acuerdo con su nivel de demanda cognitiva.

De acuerdo con el modelo, este criterio de alineamiento busca determinar si lo que se aborda en las clases y lo que se les solicita a los estudiantes, reflejado en los libros de texto, es tan exigente, cognitivamente, como lo declarado en los estándares. En sus múltiples trabajos sobre alineamiento Webb considera cuatro niveles o categorías de pensamiento que van desde el nivel más básico hasta el más complejo: Memorístico, de Procesamiento, Estratégico y Extendido.

En el Cuadro 1 se describen los niveles de la demanda cognitiva(DOK) utilizados por Webb y orientados al área de las Matemáticas:

\begin{tabular}{|c|c|}
\hline Descripciones por Nivel & Actividades Matemáticas relacionadas \\
\hline $\begin{array}{l}\text { NIVEL } 1 \\
\text { Recordar y reproducir } \\
\text { Pensamiento Memorístico: } \\
\text { Demuestra conocimiento en forma igual o casi } \\
\text { igual a como lo aprendido. }\end{array}$ & $\begin{array}{l}\text { Recuerda o reconoce hechos, definiciones o términos. } \\
\text { Aplica un algoritmo o una fórmula. } \\
\text { Identifica una figura de dos o tres dimensiones. } \\
\text { Lleva a cabo un procedimiento establecido. } \\
\text { Evalúa una expresión. } \\
\text { Resuelve un problema verbal de un paso. }\end{array}$ \\
\hline $\begin{array}{l}\text { NIVEL } 2 \\
\text { Habilidades y conceptos } \\
\text { Pensamiento de Procesamiento: } \\
\text { Demuestra conocimiento que requiere algún } \\
\text { razonamiento mental básico de ideas, conceptos y } \\
\text { destrezas, más allá de la memoria. }\end{array}$ & $\begin{array}{l}\text { Interpreta información de una gráfica. } \\
\text { Utiliza modelos para representar conceptos } \\
\text { matemáticos } \\
\text { Resuelve un problema rutinario que requiera varios } \\
\text { pasos o la aplicación de múltiples } \\
\text { conceptos. Requiere procesos mentales más allá de } \\
\text { una respuesta habitual }\end{array}$ \\
\hline $\begin{array}{l}\text { NIVEL } 3 \\
\text { Pensamiento estratégico: } \\
\text { Demuestra conocimiento basado en demanda }\end{array}$ & $\begin{array}{l}\text { Interpreta información de una gráfica compleja. } \\
\text { Explica su razonamiento cuando más de una solución } \\
\text { es posible. }\end{array}$ \\
\hline
\end{tabular}




\begin{tabular}{|c|c|}
\hline $\begin{array}{l}\text { cognitiva compleja y abstracta. } \\
\text { Requiere el razonamiento, la planificación, el uso } \\
\text { de pruebas, y un mayor nivel de pensar. }\end{array}$ & $\begin{array}{l}\text { Establece y/o justifica una conjetura. } \\
\text { Desarrolla argumentos lógicos para un concepto. } \\
\text { Utiliza conceptos e ideas para resolver problemas. } \\
\text { Lleva a cabo un procedimiento con múltiples pasos y } \\
\text { que requieren toma de decisiones. } \\
\text { Generaliza un patrón. }\end{array}$ \\
\hline $\begin{array}{l}\text { NIVEL } 4 \\
\text { Pensamiento extendido } \\
\text { Extiende su conocimiento a contextos más } \\
\text { amplios. } \\
\text { Requiere el razonamiento complejo, la } \\
\text { planificación, el desarrollo, y el pensamiento. } \\
\text { Implica hacer conexiones entre ideas y conceptos, } \\
\text { escoger entre varias alternativas para resolver un } \\
\text { problema, o aplicar los resultados de un } \\
\text { experimento en otros contextos. }\end{array}$ & $\begin{array}{l}\text { Relaciona los conceptos matemáticos con otras áreas } \\
\text { de contenido. } \\
\text { Relaciona los conceptos matemáticos con aplicaciones } \\
\text { del mundo real. } \\
\text { Aplica un modelo matemático a una situación o } \\
\text { problema. } \\
\text { Conduce una investigación que especifica un } \\
\text { problema, identifica sus pasos. } \\
\text { Recolecta datos teniendo en cuenta varias variables y } \\
\text { analiza los resultados; plantea una regla para un patrón } \\
\text { complejo y encuentra un fenómeno que exhiba ese } \\
\text { comportamiento; modela un fenómeno social con } \\
\text { varias alternativas y escoge un método para resolverlo } \\
\text { usando un modelo matemático. }\end{array}$ \\
\hline
\end{tabular}

Cuadro 1 - Niveles de Demanda Cognitiva (DOK)

Fuente: elaboración propia a partir de Webb (1997).

\section{Método}

Este estudio es de tipo descriptivo y fue realizado sobre el conjunto de estándares del bloque de Álgebra de los grados $7^{\circ}, 8^{\circ}$ y $9^{\circ}$ de la Educación Básica, de los ítems utilizados en las Pruebas Nacionales de evaluación, y de las tareas o actividades que proponen los libros de texto oficiales utilizados para la enseñanza de las Matemáticas en esos grados. La Tabla 1 resume esta información:

Tabla 1 - Cantidad de tareas, ítems y estándares de álgebra en los grados $7^{\circ}, 8^{\circ}$ y $9^{\circ}$

\begin{tabular}{cccc}
\hline Grado & Ítems en pruebas & $\begin{array}{c}\text { Actividades en } \\
\text { Libros de Texto }\end{array}$ & Estándares \\
\hline $\mathbf{7}^{\mathbf{0}}$ & 31 & 415 & 8 \\
$\mathbf{8}^{\mathbf{0}}$ & 34 & 548 & 10 \\
$\mathbf{9}^{\mathbf{0}}$ & 45 & 949 & 12 \\
Totales & 110 & 1912 & 30 \\
\hline
\end{tabular}

Fuente: elaboración propia a partir de libros de texto (EDUCACION, 2011b; 2011c; 2011d).

El estudio se realizó con la participación de veinte profesionales hondureños seleccionados por disponibilidad, todos profesores de Matemáticas con grado de licenciatura, con un promedio de 14,5 años de experiencia docente, nueve de ellos con máster en matemática educativa, tres con maestría en otra área (Física, Administración) y un doctor en educación.

En primer lugar, la tarea de los profesores consistió en clasificar tanto los estándares como los ítems propuestas en las Pruebas Nacionales de evaluación del año 2014, de acuerdo con el nivel de demanda cognitiva al que perteneciesen, siguiendo la propuesta de Webb (1997) presentada en el Cuadro 1. 
La participación de los profesores fue en calidad de Juez (J) y manifestaron su criterio asignándole el valor de 1, 2 ,3 ó 4 según el nivel DOK a cada estándar y a cada actividad o tarea. Al final se tomó como válido el valor de mayor frecuencia de aparición, su moda.

Así, por ejemplo, el estándar de séptimo grado del componente Ecuaciones y desigualdades: resuelven problemas de la vida cotidiana que impliquen ecuaciones lineales en una variable con coeficientes racionales (enteros, fracciones y decimales), fue clasificado por los jueces como estándar que pertenece al nivel DOK 3. de acuerdo con los criterios propuestos por Webb (1997), presentados en el Cuadro 1.

Dado que uno o más estándares hacen referencia a un mismo componente, se determinó el nivel DOK de cada uno de ellos, determinado por su moda, y, de esta manera, cada componente fue asociado a un nivel DOK, como se muestra en Anexo A.

Una vez clasificados los estándares, fueron clasificados, del mismo modo, los ítems de las Pruebas Nacionales de evaluación, correspondientes al bloque de Álgebra. Por ejemplo, los profesores participantes, clasificaron en el nivel DOK 1 la siguiente pregunta: Si $t=2$ ¿cuál es el valor de $3 t$ ?

Del mismo modo clasificaron como de nivel DOK 2 la siguiente: ¿Cuál es el valor de $x$ en la ecuación: $3 x-7=-x+5$ ?

El porcentaje de acuerdo entre los jueces se calculó utilizando el Coeficiente de Concordancia de Kendall ( $w$ ) (MAROZZI, 2014). Los valores obtenidos fueron de $w=0,879$ para los treinta estándares y $w=0,823$ para las actividades o tareas de Álgebra, valores que indican un alto grado de concordancia entre los profesores participantes.

Con base al criterio establecido por los profesores participantes, fueron evaluadas a continuación los ejercicios del libro de texto en los que se propusieran actividades similares. En consecuencia, actividades como las propuestas en la Figura 2, a continuación, fueron clasificadas en el nivel de demanda cognitiva 2 :

12. Resuelva las siguientes ecuaciones.
$\begin{array}{ll}\text { (1) }-1-3 x=2 x+9 & \text { (2) } 4=-3 y+13 \\ \text { (3) } 5 x-5=26+4 x & \text { (4) } 10+2 y=12+3 y \\ \text { (5) } 5+x=2 x+1 & \text { (6) } 4 x-1=2 x-15 \\ \text { (7) } 6 x+7=17 & \text { (8) } 4-3 x=4-x \\ \text { (9) }-6 x=12-2 x & \text { (10) } 6+2 x=5 x-3\end{array}$

Figura 2 - Ejercicios propuestos en el Libro de Texto $7^{\circ}$ Fuente: EDUCACIÓN (2011b, p. 82).

El mismo procedimiento fue utilizado con las 1912 actividades propuestas en los libros 
de texto.

Por último, siguiendo el criterio propuesto en el modelo Webb, se establecieron los siguientes valores para el alineamiento entre las actividades de los libros de texto y los estándares educativos (Tabla 2).

Tabla 2 - Criterio de alineamiento con base en demanda cognitiva

\begin{tabular}{lc}
\hline $\begin{array}{l}\text { \% de actividades que coinciden con el nivel } \\
\text { cognitivo del componente }\end{array}$ & Criterio de alineamiento \\
\hline $\mathbf{5 0} \%$ o más & Fuerte \\
\hline $\mathbf{4 0 \%}-\mathbf{5 0} \%$ & Débil \\
\hline Menos de $\mathbf{4 0} \%$ & Inadecuado \\
\hline
\end{tabular}

Fuente: Webb (1997).

El 50\% es un punto de corte conservador, se basa en la suposición de que una puntuación mínima de aprobación para cualquier estándar requeriría que el estudiante responda correctamente algunos ítems sobre el nivel cognitivo del estándar correspondiente (WEBB, 2005).

\section{Resultados Obtenidos}

Las Figuras 3, 4 y 5 muestran la distribución, por nivel de demanda cognitiva, de las tareas o actividades que proponen los libros de texto para cada componente de Álgebra en sus respectivos grados.

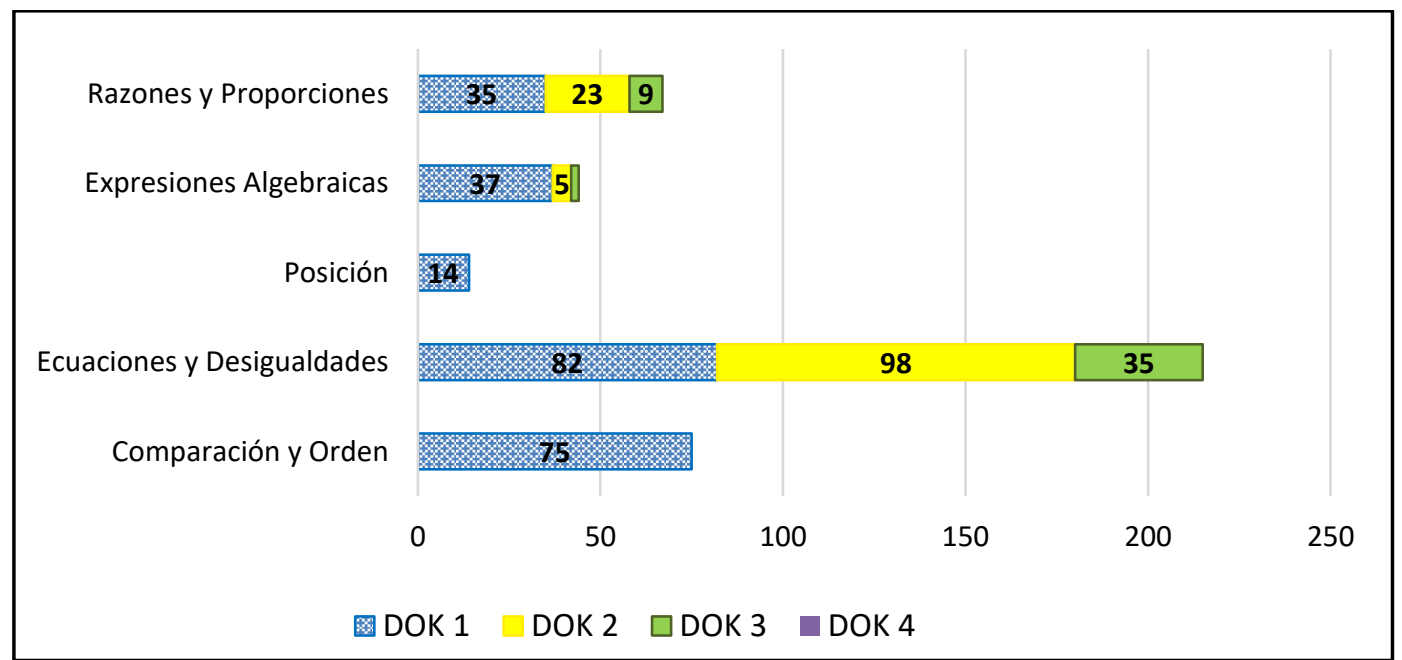

Figura 3 - Cantidad de tareas de Álgebra por nivel DOK y componente en Libros de Texto grado $7^{\circ}$ Fuente: elaboración propia.

Como puede observarse, en el libro de texto de séptimo grado no se registran actividades orientadas a que el alumno extienda sus conocimientos a contextos más amplios, es decir al nivel DOK 4, prevalecen actividades del nivel 1 que requieren reconocer hechos, aplicar un 
algoritmo o una fórmula, actividades de un paso.

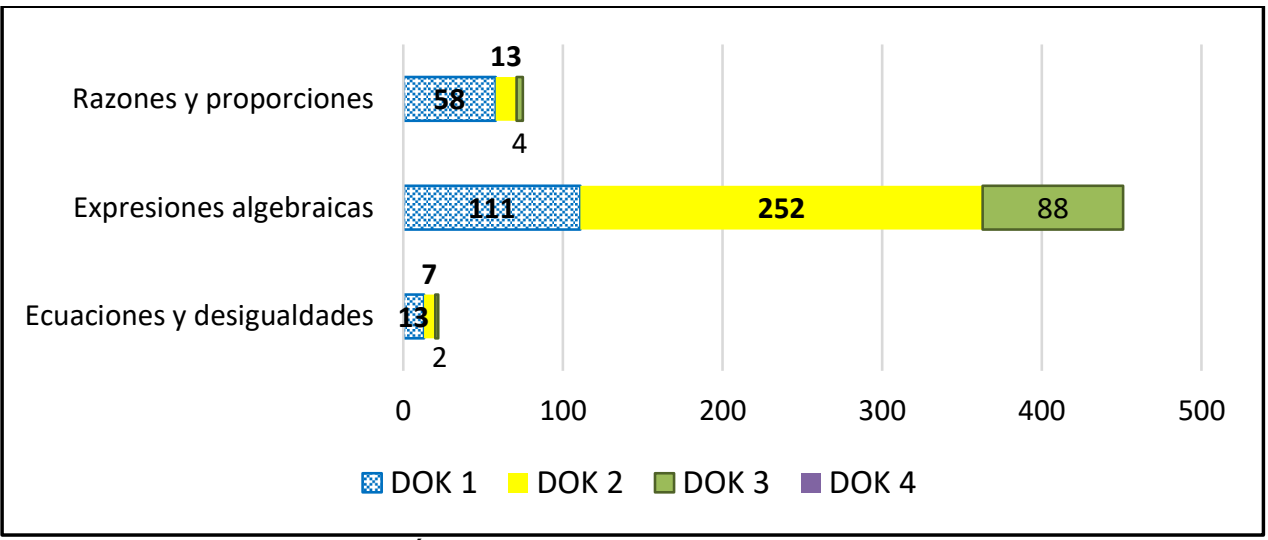

Figura 4 - Cantidad de tareas de Álgebra por nivel DOK y componente en Libros de Texto grado $8^{\circ}$ Fuente: elaboración propia.

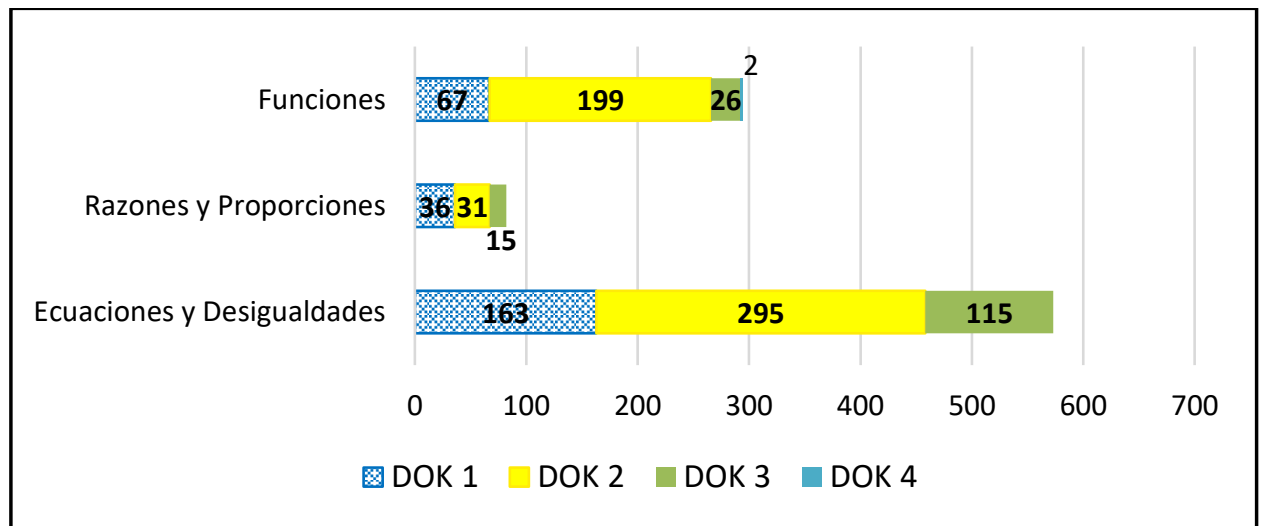

Figura 5 - Cantidad de tareas de Álgebra por nivel DOK y componente en Libros de Texto grado $9^{\circ}$ Fuente: elaboración propia.

Al igual que el grado $7^{\circ}$, en $8^{\circ}$ no se registran actividades del nivel DOK 4 , y en $9^{\circ}$ se registran tan sólo dos, pertenecientes al componente de funciones.

Las Figuras 6, 7 y 8 muestran una comparación porcentual entre estándares y actividades propuestas en los libros de texto, por grado, según su nivel de demanda cognitiva:

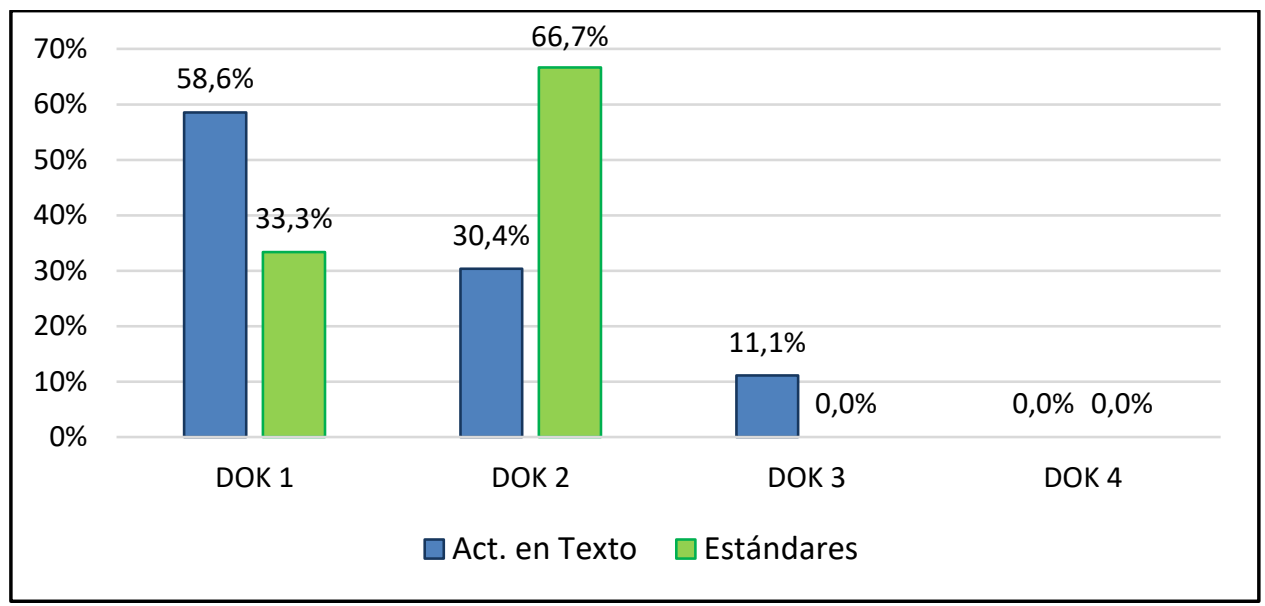

Figura 6 - Porcentajes de estándares y tareas de libro de texto por nivel DOK, $7^{\circ}$ Fuente: elaboración propia. 


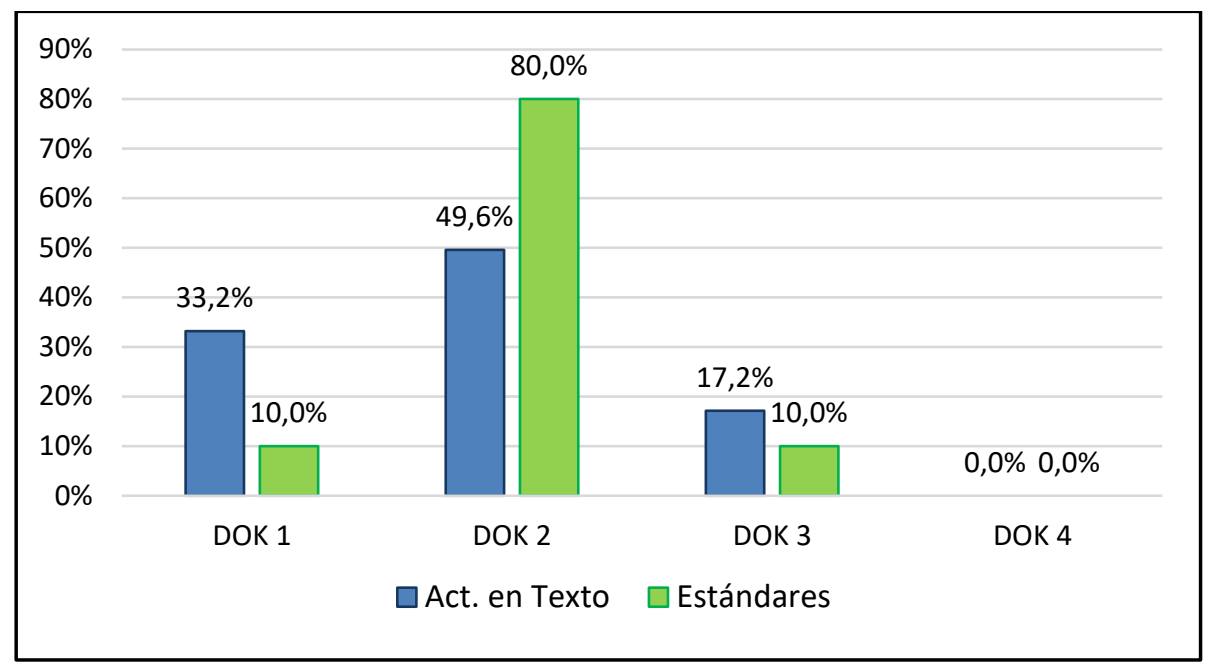

Figura 7 - Porcentajes de estándares y tareas de libro de texto por nivel DOK, $8^{\circ}$ Fuente: elaboración propia.

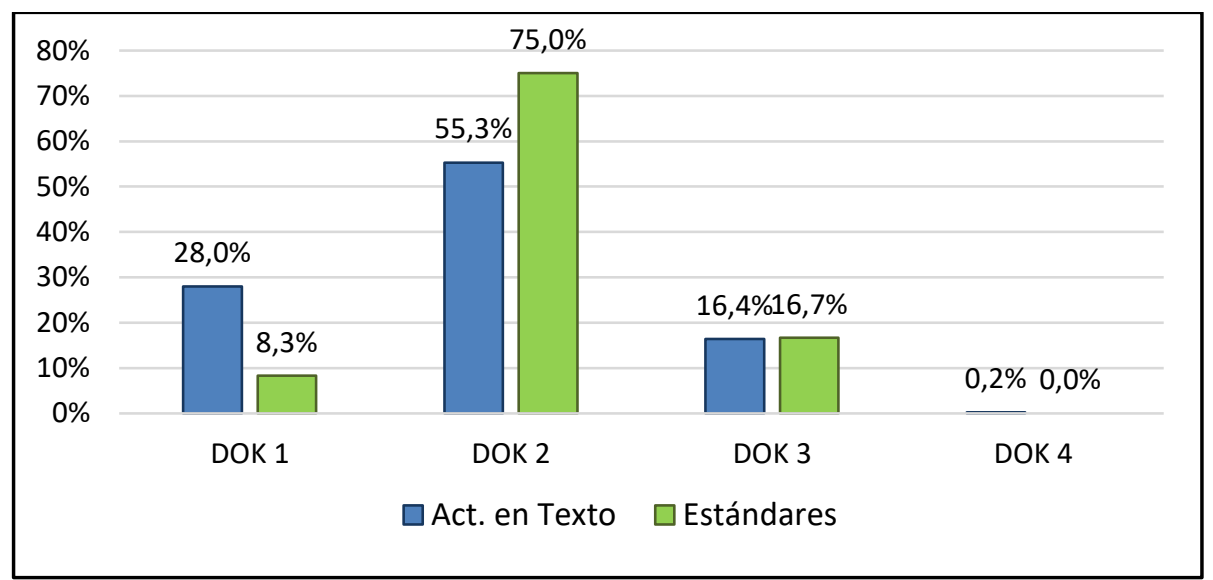

Figura 8 - Porcentajes de estándares y tareas de libro de texto por nivel DOK, $9^{\circ}$ Fuente: elaboración propia.

En las figuras anteriores se puede apreciar que no se registran estándares con un nivel de demanda cognitiva 4 y únicamente en noveno grado un pequeño porcentaje de tareas $(0,2 \%)$ corresponden a este nivel.

El alineamiento entre estándares y libros de texto, utilizando el criterio de demanda cognitiva, no exige que el porcentaje de estándares y el de las actividades o tareas que los libros de texto proponen deban coincidir totalmente en cada nivel de demanda cognitiva. Como se dijo anteriormente, el alineamiento es fuerte cuando al menos el 50\% de las actividades coinciden con el nivel DOK del componente.

Un ejemplo de aplicar el criterio que se expone en la Tabla 3 es el siguiente: en noveno grado, los siete estándares del componente Ecuaciones y desigualdades lo ubican en el nivel DOK 2, el libro de texto presenta 573 actividades relacionadas con este componente, de las cuales el 52\% (295 actividades) pertenecen también al nivel DOK 2. Esto significa que en este componente el alineamiento es fuerte. 
Siguiendo esta metodología, fueron analizadas 1912 actividades que proponen los libros de texto de Matemáticas para los grados $7^{\circ}, 8^{\circ}$ y $9^{\circ}$, y se hizo la comparación con cada uno de sus componentes. Los resultados se muestran a continuación, en las Tablas 3, 4 y 5.

Tabla 3 - Alineamiento con base en demanda cognitiva, séptimo grado

\begin{tabular}{|c|c|c|c|c|c|c|}
\hline \multirow{2}{*}{ Componente } & \multirow{2}{*}{ Nivel DOK } & \multirow{2}{*}{$\begin{array}{c}\text { Núm. de } \\
\text { actividades } \\
\text { relacionadas }\end{array}$} & \multicolumn{3}{|c|}{$\begin{array}{l}\text { Nivel de las actividades con relación con } \\
\text { los estándares }\end{array}$} & \multirow{2}{*}{$\begin{array}{l}\text { Criterio de } \\
\text { alineamiento }\end{array}$} \\
\hline & & & $\begin{array}{l}\text { Promedio } \\
\text { menor }\end{array}$ & $\begin{array}{l}\text { Promedio } \\
\text { igual }\end{array}$ & $\begin{array}{l}\text { Promedio } \\
\text { mayor }\end{array}$ & \\
\hline $\begin{array}{l}\text { Comparación } \\
\text { y orden }\end{array}$ & 2 & 75 & 100 & 0 & 0 & Inadecuado \\
\hline $\begin{array}{l}\text { Ecuaciones y } \\
\text { desigualdades }\end{array}$ & 2 & 215 & $38 \%$ & $46 \%$ & $16 \%$ & Fuerte \\
\hline Posición & 2 & 14 & $100 \%$ & 0 & 0 & Inadecuado \\
\hline $\begin{array}{l}\text { Expresiones } \\
\text { algebraicas }\end{array}$ & 2 & 44 & $84 \%$ & $11 \%$ & $5 \%$ & Inadecuado \\
\hline $\begin{array}{l}\text { Razones y } \\
\text { proporciones }\end{array}$ & 3 & 67 & $87 \%$ & $13 \%$ & $0 \%$ & Inadecuado \\
\hline
\end{tabular}

Fuente: elaboración propia.

Tabla 4 - Alineamiento con base en demanda cognitiva, octavo grado

\begin{tabular}{|c|c|c|c|c|c|c|}
\hline \multirow{2}{*}{ Componente } & \multirow{2}{*}{ Nivel DOK } & \multirow{2}{*}{$\begin{array}{c}\text { Núm. de } \\
\text { actividades } \\
\text { relacionadas }\end{array}$} & \multicolumn{3}{|c|}{$\begin{array}{l}\text { Nivel de las actividades con relación con } \\
\text { los estándares }\end{array}$} & \multirow{2}{*}{$\begin{array}{l}\text { Criterio de } \\
\text { alineamiento }\end{array}$} \\
\hline & & & $\begin{array}{c}\text { Promedio } \\
\text { menor }\end{array}$ & $\begin{array}{c}\text { Promedio } \\
\text { igual }\end{array}$ & $\begin{array}{c}\text { Promedio } \\
\text { mayor }\end{array}$ & \\
\hline $\begin{array}{l}\text { Ecuaciones y } \\
\text { desigualdades }\end{array}$ & 2 & 22 & $59 \%$ & $32 \%$ & $9 \%$ & Inadecuado \\
\hline $\begin{array}{l}\text { Expresiones } \\
\text { algebraicas }\end{array}$ & 2 & 451 & $25 \%$ & $56 \%$ & $20 \%$ & Fuerte \\
\hline $\begin{array}{l}\text { Razones y } \\
\text { proporciones }\end{array}$ & 3 & 75 & $95 \%$ & $5 \%$ & $0 \%$ & Inadecuado \\
\hline
\end{tabular}

Fuente: elaboración propia.

Tabla 5 - Alineamiento con base en demanda cognitiva, noveno grado

\begin{tabular}{lcccccc}
\hline Componente & Nivel DOK & $\begin{array}{c}\text { Núm. de } \\
\text { actividades }\end{array}$ & Nivel de las actividades con relación con & Criterio de \\
relacionadas & & $\begin{array}{c}\text { Promedio } \\
\text { menor }\end{array}$ & $\begin{array}{c}\text { Promedióndares } \\
\text { igual }\end{array}$ & $\begin{array}{c}\text { Promedio } \\
\text { mayor }\end{array}$ & alineamiento \\
\hline $\begin{array}{l}\text { Ecuaciones y } \\
\begin{array}{l}\text { Desigualdades } \\
\text { Razones y }\end{array}\end{array}$ & 2 & 573 & $28 \%$ & $51 \%$ & $20 \%$ & Fuerte \\
$\begin{array}{l}\text { Proporciones } \\
\text { Funciones }\end{array}$ & 3 & 82 & $82 \%$ & $18 \%$ & $0 \%$ & Inadecuado \\
\hline & 2 & 294 & $23 \%$ & $68 \%$ & $10 \%$ & Fuerte \\
\hline
\end{tabular}

Como puede deducirse al revisar cada una de las tablas, el alineamiento a nivel de demanda cognitiva entre estándares y libros de texto para el bloque de Álgebra, en estos tres grados de la Educación Básica de Honduras, no es totalmente adecuado, pues en cada grado encontramos componentes con un alineamiento inadecuado, siendo $7^{\circ}$ el grado que más presenta esta situación.

En los tres grados, muy pocas actividades que se proponen en los libros de texto superan el nivel cognitivo que suponen los estándares respectivos, e incluso en algunos casos no se 
identifican actividades con un nivel de exigencia superior.

\section{Discusión y Conclusiones}

La principal reflexión a que nos deben llevar los resultados de este estudio es que, si el libro de texto tiene una importante influencia en las prácticas docentes, conviene plantearse una revisión profunda de los libros de texto para la enseñanza de las Matemáticas en la educación básica de Honduras o de sus estándares educativos.

Un criterio para considerar debe ser el de demanda cognitiva, pues se muestra un bajo porcentaje de alineamiento o coherencia en los grados analizados y es, como lo plantea Smith y Stein (1998), el elemento de mayor influencia en el desarrollo de la capacidad de pensar, razonar y resolver problemas, por lo que es importante proponer tareas de alto nivel cognitivo en todas las lecciones.

Si el objetivo final es que los estudiantes desarrollen la capacidad de pensar, razonar y resolver problemas es importante comenzar con tareas de alto nivel cognitivo (SMITH; STEIN, 1998). En esa línea, resulta interesante hacer una revisión exhaustiva de los recursos de apoyo para la implementación del CNEB en Honduras, específicamente en el área de Matemáticas, con la idea de asegurar que los libros de texto, las evaluaciones estandarizadas y las capacitaciones docentes propongan tareas de suficiente nivel cognitivo.

Al analizar la propuesta de abordar el bloque de Álgebra en los libros de texto encontramos que no existen actividades o tareas que trasciendan a un nivel de demanda cognitiva 4, sobre pensamiento extendido que permita al estudiante a relacionar conceptos matemáticos con otras áreas de contenido, con aplicaciones del mundo real o que motiven a desarrollar un modelo matemático ante una situación o problema.

Con la información obtenida se confirma que con el uso de los libros de texto oficiales para enseñar Matemáticas en los grados $7^{\circ}, 8^{\circ}$ y $9^{\circ}$, en el bloque de Álgebra se promueve el razonamiento mental básico de ideas, mientras que muy poco se trasciende al nivel 3 que implica Pensamiento Estratégico y la habilidad para demandas cognitivas más complejas y abstractas, mientras que no existen elementos orientados al razonamiento complejo en los conceptos algebraicos, es decir nivel 4.

La conclusión clave de este estudio es que la coherencia entre estándares y libros de texto no es adecuada, pues los estándares tienen una mayor exigencia cognitiva que las actividades que proponen los libros de texto.

Con los resultados del presente estudio se puede sugerir que, para próximas ediciones 
de los libros de texto, se incluyan actividades de mayor demanda cognitiva, esto además de incrementarles el nivel de exigencia hará que haya un mejor alineamiento entre estas dos piezas claves del currículo.

La aplicación del modelo de Webb ha sido utilizada para evaluar el alineamiento entre estándares y evaluación, pero no encontramos referencias a estudios que incidan en el alineamiento entre estándares y libros de texto. El currículum y la instrucción en el aula deben estar alineados con los estándares y la evaluación, pero si este alineamiento no está presente en las actividades propuestas a los estudiantes, particularmente en los libros de texto utilizados, éstos no pueden adquirir el conocimiento y las habilidades que necesitan para lograr los estándares (LINN; HERMAN, 1997).

La metodología de análisis utilizada puede resultar muy útil para extender el proceso de valoración del alineamiento entre las actividades docentes y los estándares educativos a todos los grados y bloques de la matemática. De este modo, un mejor alineamiento se reflejará en mejores resultados de la evaluación.

\section{Referencias}

CARR, J.; HARRIS, D. Succedin With Standars, Linking Curriculum, Assessment, and action Planning. Alexandria, Virginia, USA: Association for Supervision; Curriculum Deve; Edition Unstated edition, 2001.

EDUCACIÓN, SECRETARÍA de. Diseño Curricular Nacional para la Educación Básica. Tegucigalpa, Honduras: SE, 2003.

EDUCACIÓN, SECRETARÍA de. Estándares Educativos Nacionales Español y Matemáticas 1ro a 11mo grado. Tegucigalpa: SE, 2011a.

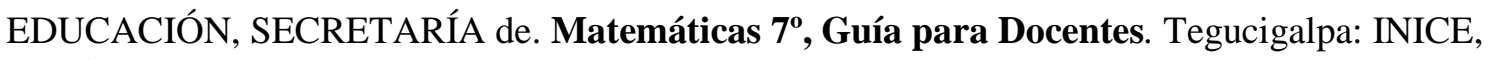
$2011 b$.

EDUCACIÓN, SECRETARÍA de. Matemáticas $\mathbf{8}^{\boldsymbol{o}}$, Guía para Docentes. Tegucigalpa: INICE, 2011c.

EDUCACIÓN, SECRETARÍA de. Matemáticas 9 $^{\circ}$, Guía para Docentes. Tegucigalpa: INICE, 2011d.

EDUCACIÓN, SECRETARÍA de. Informe Nacional de Desempeño Académico: Español y Matemáticas 1ro a 9no grado 2016. Tegucigalpa: SE-Proyecto MIDEH, 2017.

HERMAN, J.; WEBB, N.; ZUNIGA, S. Measurement Issues in the Alignment of Standards and Assessments: A Case Study. Los Angeles, LA: Center for the Study of Evaluation National Center for Research on Evaluation, Standards, and Student Testing Graduate School of Education ; Information Studies University of California, 2005.

JICA. JICA firma acuerdo de cooperación técnica con Educación y la UPNFM. 2015. Disponible en: 〈http://www.jica.go.jp/honduras/espanol/office/topics/150714_01.html>. Acceso en: 15 mar. 2017. 
LEBRUN, J.; MORESOLI, C.; HASNI, A. Funciones de los textos escolares: Un análisis comparativo del contexto de Quebec y Ontario. Revista Iberomericana de Evaluación Educativa, Madrid, p. 8197, 2012.

LINN, R.; HERMAN, J. La Evaluación impulsada por estándares: Problemas técnicos y políticos en la medición del progreso de la escuela y los estudiantes. Programa de Promoción de la Reforma Educativa en América Latina y el Caribe, 1997. p. 1-35.

LLINARES, S. Agendas de Investigación en Educación Matemática en España. En: LUENGO, R. et al. Investigación en educación MATEMATICA XII. Badajoz: Facultad de Educación, Universidad de Extremadura, 2008. p. 25-53.

MAROZZI, M. Testing for concordance between several criteria. Journal of Statistical Computation and Simulation, Taylor and Francis Online, v. 9, n. 84, p. 1843-1850, 2014.

MARTÍNEZ, J.; RODRÍGUEZ, J. El currículum y el libro de texto. Una dialéctica siempre abierta. En: SACRISTÁN, J. J. Saberes e incertidumbres sobre el currículum. Madrid: Ediciones Morata, 2010. p. 246-268.

MARTONE, A.; SIRECI, S. Evaluating Alignment Between Curriculum, Assessment, and Instruction. Review of Educational Research, SAGE journals, n. 79, p. 1332-1361, 2009.

NCTM. Principios y Estándares para la Educación Matemática. Sevilla: Sociedad Andaluza de Educación Matemática Thales, 2000.

PENALVA, M.; LLINARES, S. Tareas matemáticas en la educación secundaria. En: GOÑI, J. (Coord.). Didáctica de las Matemáticas. Barcelona: GRAO, 2011. p. 27-52.

SMITH, M.; STEIN, M. Selecting and Creating Mathematical Tasks: From Research to Practice. Mathematical Teaching in the Middle School, ERIC, n. 3, p. 344-350, 1998.

WEBB, N. Criteria for alignment of expectations and assessments in mathematics and science education. Council of Chief State School Officers and National Institute for Science Education. Madisson, WI: Wisconsin Center for Education Research, University of Wisconsin, 1997.

WEBB, N. An Analysis of the Alignment Between Mathematics Standards and Assessments for Three States. Madison: Wisconsin Center for Education Research, 2002.

WEBB, N. Web Alignment Tool, Training Manual. Wisconsin: Wisconsing Center for Education Research, 2005.

Submetido em 27 de Setembro de 2017. Aprovado em 19 de Abril de 2018. 


\section{Anexo A}

Cuadro 2 - Consenso sobre el Nivel de Demanda Cognitiva (DOK), $7^{\circ}$

\begin{tabular}{|c|l|c|}
\hline \multicolumn{2}{|c|}{ Componentes y Estándares de Séptimo grado } \\
\hline $\mathbf{N}^{\mathbf{0}}$ & \multicolumn{1}{|c|}{ COMPONENTE/ESTANDAR } & DOK \\
\hline $\mathbf{1}$ & COMPARACION Y ORDEN & $\mathbf{2}$ \\
\hline 1.1 & Comparan y ordenan números racionales (enteros, fracciones y decimales). & 2 \\
\hline $\mathbf{2}$ & ECUACIONES Y DESIGUALDADES & $\mathbf{2}$ \\
\hline 2.1 & $\begin{array}{l}\text { Resuelven ecuaciones lineales en una sola variable con coeficientes racionales (enteros, } \\
\text { fracciones y decimales). }\end{array}$ & 2 \\
\hline 2.2 & $\begin{array}{l}\text { Resuelven problemas de la vida cotidiana que impliquen ecuaciones lineales en una } \\
\text { variable con coeficientes racionales (enteros, fracciones y decimales). }\end{array}$ & 3 \\
\hline $\mathbf{3}$ & POSICION & $\mathbf{2}$ \\
\hline 3.1 & Representan números racionales (enteros, fracciones y decimales) en la recta numérica. & 2 \\
\hline $\mathbf{4}$ & EXPRESIONES ALGEBRAICAS & $\mathbf{2}$ \\
\hline 4.1 & $\begin{array}{l}\text { Usan expresiones algebraicas para escribir matemáticamente frases de la vida cotidiana } \\
\text { y viceversa. }\end{array}$ & 2 \\
\hline 4.2 & Calculan el valor numérico de una expresión algebraica usando números racionales. & 2 \\
\hline $\mathbf{5}$ & RAZONES Y PROPORCIONES & $\mathbf{3}$ \\
\hline 5.1 & Resuelven problemas que involucran proporcionalidad directa aplicando la regla de tres. & 3 \\
\hline 5.2 & Resuelven problemas que involucran proporcionalidad inversa aplicando la regla de tres. & 3 \\
\hline
\end{tabular}

Fuente: elaboración propia

Cuadro 3 - Consenso sobre el Nivel de Demanda Cognitiva (DOK), $8^{\circ}$

\begin{tabular}{|c|l|c|}
\hline \multicolumn{2}{|c|}{ COMPONentes y Estándares de Octavo grado } \\
\hline $\mathbf{N}^{\mathbf{0}}$ & \multicolumn{1}{|c|}{ COMTE/ESTANDAR } & DOK \\
\hline $\mathbf{1}$ & ECUACIONES Y DESIGUALDADES & $\mathbf{2}$ \\
\hline 1.1 & Despejan una variable en una fórmula dada. & 2 \\
\hline $\mathbf{2}$ & EXPRESIONES ALGEBRAICAS & $\mathbf{2}$ \\
\hline 2.1 & Identifican, clasifican, ordenan y completan polinomios. & 2 \\
\hline 2.2 & Realizan adiciones y sustracciones con polinomios. & 2 \\
\hline 2.3 & Realizan multiplicaciones de polinomios con coeficientes enteros. & 2 \\
\hline 2.4 & Realizan divisiones de polinomios con coeficientes enteros. & 2 \\
\hline 2.5 & Factorizan completamente polinomios en el conjunto de los números racionales. & 2 \\
\hline 2.6 & Simplifican expresiones racionales algebraicas. & 2 \\
\hline 2.7 & Realizan operaciones básicas con expresiones racionales algebraicas. & $\mathbf{3}$ \\
\hline $\mathbf{3}$ & RAZONES Y PROPORCIONES & 3 \\
\hline 3.1 & $\begin{array}{l}\text { Resuelven problemas de la vida cotidiana utilizando porcentajes (menor que 1 y mayor } \\
\text { que 100). }\end{array}$ \\
\hline
\end{tabular}


3.2 Usan la calculadora para encontrar el tanto por ciento de un número

2

Fuente: elaboración propia

Cuadro 4 - Consenso sobre el Nivel de Demanda Cognitiva (DOK), $9^{\circ}$

\begin{tabular}{|c|c|c|}
\hline \multicolumn{3}{|c|}{ Componentes y Estándares de Noveno grado } \\
\hline $\mathbf{N}^{\mathbf{o}}$ & COMPONENTE/ESTANDAR & DOK \\
\hline 1 & ECUACIONES Y DESIGUALDADES & 2 \\
\hline 1.1 & Encuentran la solución de ecuaciones cuadráticas en una sola variable. & 2 \\
\hline 12 & $\begin{array}{l}\text { Resuelven problemas de la vida cotidiana que impliquen ecuaciones cuadráticas en una } \\
\text { sola variable. }\end{array}$ & 3 \\
\hline 1.3 & $\begin{array}{l}\text { Resuelven sistemas de dos ecuaciones lineales en dos variables aplicando los métodos } \\
\text { de sustitución, igualación y eliminación. }\end{array}$ & 2 \\
\hline 1.4 & $\begin{array}{l}\text { Resuelven problemas de la vida cotidiana que impliquen la solución de un sistema de dos } \\
\text { ecuaciones lineales en dos variables. }\end{array}$ & 3 \\
\hline 1.5 & Resuelven inecuaciones lineales en una variable con coeficientes racionales. & 2 \\
\hline 1.6 & $\begin{array}{l}\text { Resuelven problemas de la vida cotidiana que impliquen inecuaciones lineales en una } \\
\text { variable con coeficientes racionales. }\end{array}$ & 2 \\
\hline 1.7 & $\begin{array}{l}\text { Usan la computadora o calculadora para comprobar las soluciones de un sistema de dos } \\
\text { ecuaciones }\end{array}$ & 2 \\
\hline 2 & RAZONES Y PROPORCIONES & 3 \\
\hline 2.1 & $\begin{array}{l}\text { Resuelven problemas de la vida cotidiana utilizando el tanto por ciento incluyendo } \\
\text { descuentos, impuestos, interés simple y compuesto. }\end{array}$ & 3 \\
\hline 3 & FUNCIONES & 2 \\
\hline 3.1 & $\begin{array}{l}\text { Determinan la ecuación de una recta (dados: dos puntos, un punto y la pendiente, un } \\
\text { punto y la ecuación de una recta paralela o perpendicular). }\end{array}$ & 2 \\
\hline 3.2 & Identifican, interpretan y grafican funciones lineales. & 2 \\
\hline 3.3 & Resuelven problemas de la vida cotidiana usando las funciones lineales. & 3 \\
\hline 3.4 & $\begin{array}{l}\text { Determinan las variables dependientes e independientes en situaciones de la vida } \\
\text { cotidiana. }\end{array}$ & 2 \\
\hline
\end{tabular}

Fuente: elaboración propia 